\title{
The Care of Central Venous Catheters in the Oncopediatric Department
}

\author{
Zsuzsanna Erzsébet Papp¹, Mária-Adrienne Horváth¹, Nora Rat², Lucian Băilă3 \\ 1 Department of Pediatrics, County Emergency Clinical Hospital, University of Medicine and Pharmacy, Tîrgu Mureș, Romania \\ 2 Clinic of Cardiology, University of Medicine and Pharmacy, Tîrgu Mureș, Romania \\ 3 Department of Anesthesy and Intensive Therapy, County Emergency Clinical Hospital, Tîrgu Mureș, Romania
}

\section{CORRESPONDENCE}

\section{Zsuzsanna Erzsébet Papp}

Str. Gheorghe Marinescu nr. 38

540139 Tîrgu Mureș, Romania

Tel: +40 265214411

E-mail: pappzsuzsi@free-mail.hu

\section{ARTICLE HISTORY}

Received: 5 May, 2016

Accepted: 30 May, 2016
Mária-Adrienne Horváth • Str. Gheorghe Marinescu nr. 38, 540139 Tîrgu Mureș, Romania. Tel: +40 265214 411. E-mail: adigyer1@yahoo.com

Nora Rat • Str. Gheorghe Marinescu nr. 38, 540139 Tîrgu Mureș, Romania. Tel: +40 265215 551. E-mail: ratnora@gmail.com

Lucian Băilă • Str. Gheorghe Marinescu nr. 38, 540139 Tîrgu Mureș, Romania. Tel: +40 265215 551. E-mail:

lucian.baila@gmail.com

\begin{abstract}
Introduction: Childhood cancer, with its major psycho-social and health impact, needs longterm chemotherapy. Increasing the intensity of treatment results in improved outcomes of hematological malignancies and solid tumors. As cytostatics have a vascular irritating effect and multiple peripheric venous punctures cause pain, insertion of a long-term central venous catheter (CVC) during chemotherapy is often necessary. Materials and methods: All pediatric patients (aged below 18 years) with hematologic and malignant pathologies who underwent CVC insertion at the Pediatric Hemato-oncology Department of the County Emergency Clinical Hospital of Tîrgu Mureș in the February 2014 - May 2016 period were enrolled in the study. Results: We recorded 24 cases who received central venous catheters, out of which 14 patients received tunneled CVC, 7 patients port CVC, and 3 patients received initially tunneled CVC which was changed with port CVC. Tunneled catheters were preserved in average for 186.06 days and portacaths for 256.6 days. For infection prophylaxis and treatment every child received adequate antibiotic medication. Conclusions: The use of central lines in pediatric hemato-oncology is accessible and benefic not only for increasing patient comfort, but also to ease the nurses' work, who are often overburdened.
\end{abstract}

Keywords: child, cancer, central venous catheter

\section{INTRODUCTION}

Childhood cancer with its major psycho-social and health impact needs longterm chemotherapy. Increasing the intensity of treatment results in improved outcomes of hematological malignancies and solid tumors. Cytostatics have a vascular irritating effect, multiple peripheric venous punctures cause pain, and children have an inadequate peripheral venous vascular system, therefore during the cytostatic treatment it might be necessary to insert a long-term central venous catheter (CVC) to assure central access for basic and supportive treatment. ${ }^{1}$

Three central venous catheter types are used in pediatric hemato-oncology: totally implantable catheters (portacaths), tunneled externalized catheters (Broviac ${ }^{\oplus}$, Hickman $^{\circ}$ catheters) and peripherally inserted central catheters. ${ }^{2}$ 
The use of central venous catheters is essential for pediatric patients who require hemodialysis, parenteral nutrition, chemotherapy, or other medications. International guidelines are established for the adequate use of CVC. Indications consist of chemotherapy and preparations for bone marrow transplantation, hemodialysis, hemofiltration, antibiotic or/and antifungal treatment, central venous pressure measurement, irritating medication administration, difficult peripheric venous access, parenteral nutrition and continuous pain-management. Absolute contraindication is systemic sepsis, while relative contraindications are represented by local cellulitis and thrombocytopenia. ${ }^{1,3}$

Before insertion it is important to obtain anamnestic data regarding contrast substance allergy and to perform basic laboratory investigations (Leucocyte count, Hgb, Thrombocytes, Coagulogram). It is also highly recommended that the patient does not eat 4-6 hours before the intervention. The insertion of portacaths and tunneled catheters are performed under general anesthesia. Major bleeding complications during insertion are extremely rare when the patient is correctly monitored. By the time of the intervention, the first dose of prophylactic antibiotic is already administered. ${ }^{4}$

Intraoperative complications are: pneumothorax, pulmonary embolism, haemorrhage; long-term complications can be: migration, infections, thrombosis, mechanical complications and fibrin assemblage around the CVC.

Parenteral nutrition (PN) and external CVC are associated with an increased risk of infection and occlusion complication, but their interaction is unknown. The increase of risk is higher in children with ports: a 40 - and 10 -fold increase in infection risk and occlusion. Oncopediatric patients who need PN should have external CVC inserted. ${ }^{5}$

All mechanical, infective, or thrombotic complications favor severe life-threatening events, result in hospitalization prolongation with interrupted medical treatment, higher morbidity and mortality, and extra costs. ${ }^{6}$ In these cases, the non-functional central line is simply removed and a new catheter needs to be inserted. ${ }^{7}$

A multidisciplinary, best-practice central line maintenance care guideline reduces central line-associated blood stream infection rates in hospitalized pediatric oncology patients. ${ }^{8}$ Care principles for preventing infections differ in function of catheter type. Infrequent manipulation is required, and every time we open the catheter we have to respect aseptic rules. It is necessary to record every bandage, flushing, and changing of the bionector, and have it signed by the person who performed it.

\section{MATERIALS AND METHODS}

Our purpose was to assess CVC complications in a pediatric hemato-oncologic department.

All pediatric patients (age below 18 years) with hematologic and malignant pathologies who underwent CVC insertion at the Pediatric Hemato-oncology Department of the County Emergency Clinical Hospital of Tîrgu Mureș in the January 2013 - May 2016 period were enrolled in the study. Primary diagnosis, catheter type, complications, and reasons for removal of the device were recorded.

The study has been carried out in accordance with the code of ethics of the World Medical Association's Declaration of Helsinki. All patients consented with the inclusion in this study, and the study protocol was approved by the institution.

\section{RESULTS}

In this period, we treated 28 children with intense chemotherapy (11 female, 17 male), aged between 9 months and 18 years (mean age 7.20 years). The majority of the pathology was pre-B cell acute lymphoblastic leukemia - ALL (Figure 1). In our department we have experience with portacaths and tunneled catheters.

Out of these patients, 24 received central venous catheters: 14 patients tunneled CVC, 7 patients port CVC, and 3 patients initially tunneled CVC which was changed with port CVC (Figure 2).

One 17-year-old patient with Hodgkin lymphoma and one 3-year-old girl with Wilms tumor had no CVC because their cytostatic treatment rhythm did not permit it. One 5 -year-old male patient with Wilms tumor had left to another oncology center after the preoperative chemotherapy and received the central line there, and one 2.4-year-old

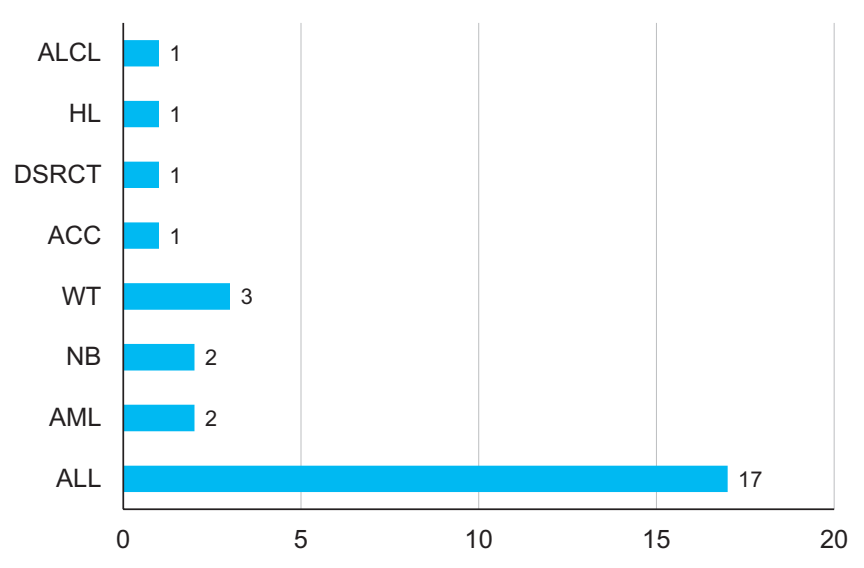

FIGURE 1. Pathology distribution 


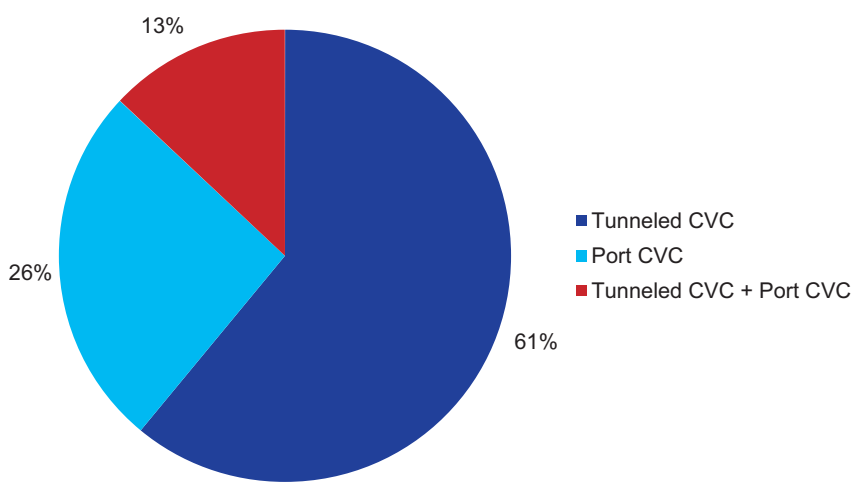

FIGURE 2. Types of inserted CVC

patient was newly diagnosed and she had no proper hematological parameters yet for the intervention.

The period between the diagnosis and the insertion of the catheter varied between 7 and 45 days (mean of 12 days). Tunneled catheter type in our centre was Hickman or Broviac, portacatheters were from Bbraun and Vygon with proper dimension for the patient age.

Tunneled catheters were preserved for 186.06 days, and portcaths for 256.6 days on average.

In 3 cases tunneled CVC were changed to portacaths. A 5.5-year-old high risk ALL female patient accidentally extracted the catheter 15 days after the insertion, because it tangled to her feet in a moment of negligence. In the case of a 15-year-old male ALC Lpatient the tunneled catheter line had to be changed because the tip of the catheter reached into the internal jugular vein. The patient had a vascular malformation, which caused the following incident: after the first course of chemotherapy he complained of extremely aggressive headache and dizziness, so we decided to remove the central line and to change it to portacath. The echo-guided insertion was difficult, but finally successful. A 12-year-old female Wilms tumor patient was treated with tunneled catheter, and after finalizing the treatment this central line was extracted. After 4 months we observed a pulmonary metastasis, and we decided that a portacath needs to be inserted to continue the chemotherapy.

We had 6 cases of incidences at the moment of insertion. Multiple unsuccessful punctures at a 17-year-old ALL female patient caused iatrogenic pneumothorax. The clinical signs were acute pain, cough, dyspnoea. She was admitted to the Department of Surgery for 10 days and was treated with continuous aspiration. In two cases of ALL patients (1.4-year-old girl, 2.4-year-old boy) we observed a hemorrhagic incident after the insertion, which was treated with fresh frozen plasma and etamsilat. In case of a 4.5-year-old ALL girl we observed a subcutaneous hematoma 2 days after the implantation, which spontaneously resorbed in 5 days (Figure 3A).

In the case of a 9-month-old male child with high risk ALL at the moment of insertion the tip of the catheter plied, the portacath would function only under pressure, therefore IV medication needed to be administered with an infusion pump.

The vascular malformation of a 14-year-old ALCL male patient permitted the tip of the tunneled catheter to reach into the internal jugular vein.

Late-term mechanical complications were observed in 5 cases: 1 thrombosis (14-year-old ACC girl), resolved with extraction-reinsertion of a new tunneled catheter and antithrombotic treatment with Clexane; 1 case of deteriorated bionector; 1 case of occluded tunneled catheter (9-yearold female patient with NB) at the end of chemotherapy,
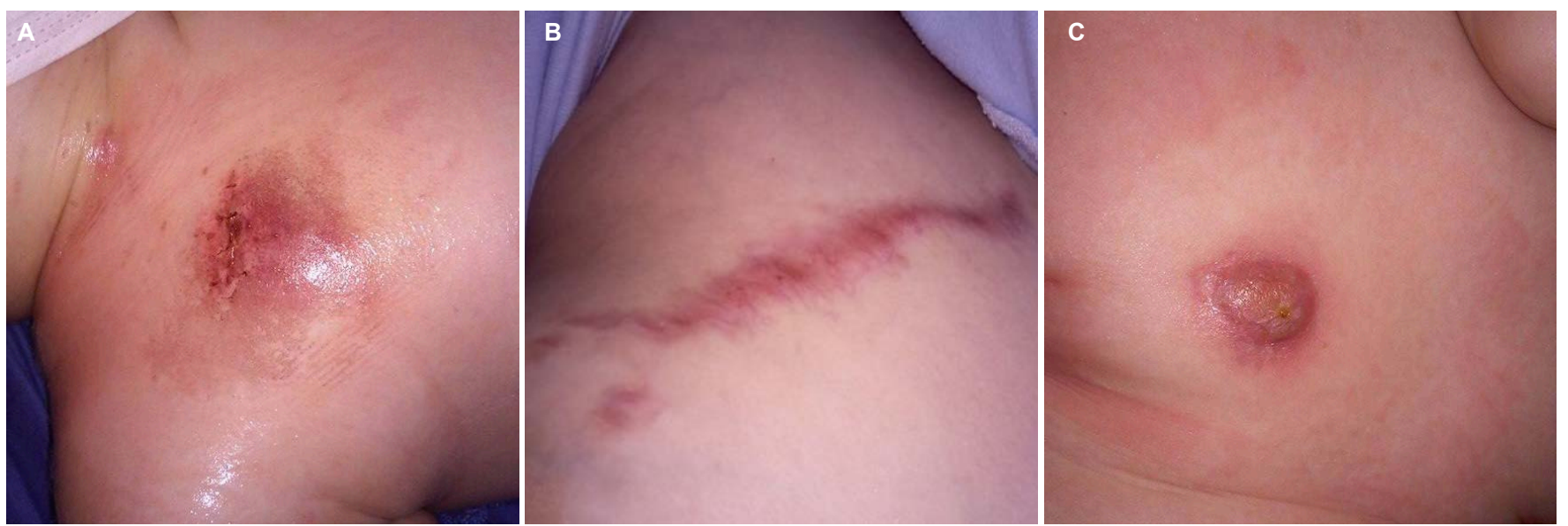

FIGURE 3. Skin modifications at the site of catheter insertion. A - Hematoma after insertion of a port catheter. $\mathbf{B}-$ The scar after the plastic surgery following catheter-associated cellulitis. $\mathbf{C}$ - Necrosis at the site of catheter insertion. 

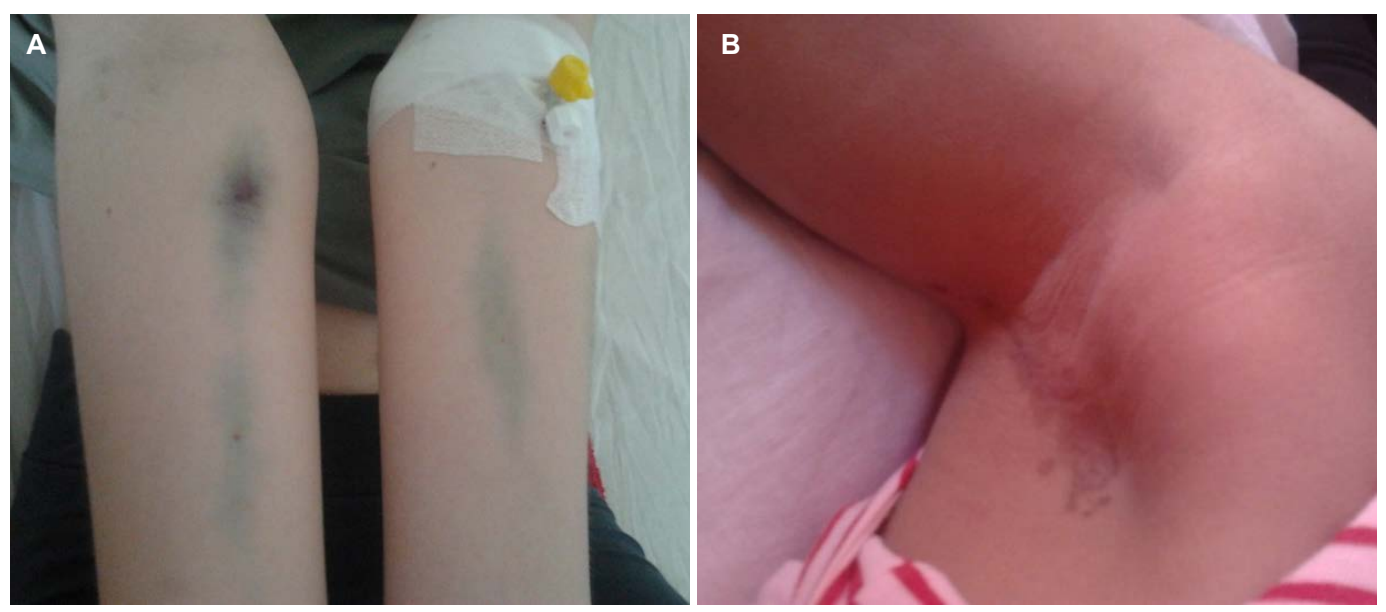

FIGURE 4. A - Echimosis at the thrombocytopenic patient. B - irritating effect on peripheral veins.

which was removed afterwards; another spontaneous obstruction of a port catheter (1.5-year-old ALL girl) which was permeabilized with intense flushing with heparine and saline solution. The last incidence was the accidental extraction of a tunneled catheter by a 5.5-year-old high risk ALL patient, afterwards she received a portacath and we preserved it after 490 days.

We had 2 infectious incidences in the aplastic period. Both of them occurred in the context of multiple skin and mucosal infections, which finally affected the insertion zone of the central line. In case of a 10-year-old boy with standard risk ALL, despite of large spectrum antibiotherapy the lesion extended, the situation required the removal of the tunneled catheter, plastic surgery (Figure 3B), and only after 3 weeks he received another tunneled catheter on the other side.

The other cellulitis (5-year-old ALL girl) responded to large spectrum IV antibiotherapy. In both cases the hemoculture was negative.

In 2 cases we observed aseptic cutaneous necrosis above the portacaths, which required the removal of the central line. In case of a14-year-old ALCL boy this happened at the end of the cytostatic protocol, but in the case of a 1-yearold ALL patient (Figure 3C), this incident came after only 3 months of intense cytostatic treatment, so we implanted an other portacath after removing the old one.

\section{DISCUSSIONS}

In our study, pathology distribution respected the literature, ALL was the most common childhood malignancy. ${ }^{9}$

Peripheric vein punctures cause pain and echimosis in the thrombocytopenic patient (Figure 4A), and cytostatic treatment has an irritating effect on peripheral veins (Fig- ure 4B). These two facts justify the use of CVC in pediatric oncology.

New types of CVCs are shown in Figure 5. The tunneled catheter is a thin, long tube made of flexible, silicone rubber. It is surgically inserted into one of the main blood vessels (internal jugular vein or vena subclavia). The catheter is going through a tunnel under the skin and openings to the lumen stick out of the skin on the chest.

The port catheter is a small plastic or metal drum with a catheter going into a large vein. It is inserted under the skin of the chest during surgery and needs special needles stuck through the skin into the septum, to use the port. The benefit of the port catheter over the tunneled CVC is that it can be kept for a longer period, it cannot get tangled and pulled out accidentally, the patient can have a whole body bath (tunneled CVC allows a partial bath only), in the medication-free period it needs to be flushed only once a month (tunneled CVC once a week).

After confirming that the patient has no contrast allergy, has a relatively normal coagulation and a platelet count greater than $50,000 / \mu \mathrm{L}$, preparations for insertion can be done. The patient is fasting for 4-6 hours before the procedure, which is performed in general anesthesia. Intraoperative antibiotic prophylaxis is recommended. Surgical technique and good anatomic landmarks are important in correct localization. ${ }^{10}$

Intraoperative complications can be represented by pneumothorax, air embolism, persistent bleeding from the insertion site and pulmonary embolism. ${ }^{11}$

Delayed complications are represented by malpositioning or migration. ${ }^{12,13}$ Venous thrombosis (treated by line removal and anticoagulation $)^{14}$ or mechanical failure can occur, and fibrin can accumulate around the line, obstructing aspiration and leading to infection..$^{15}$ 

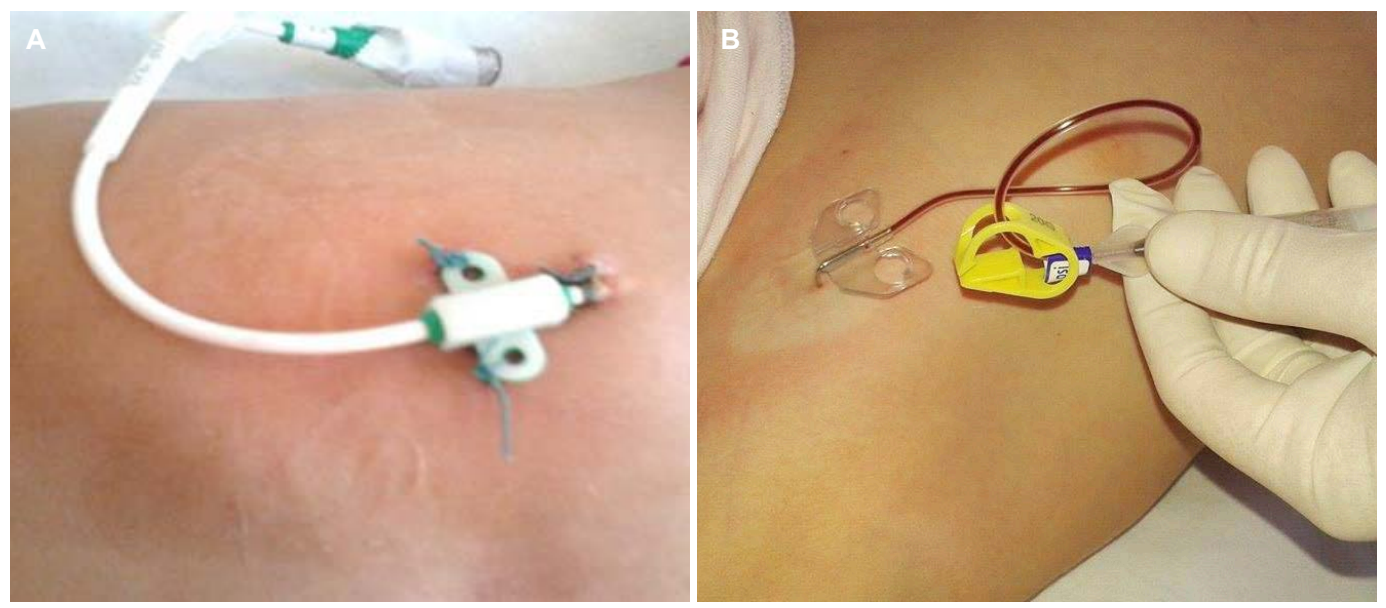

FIGURE 5. New types of catheters. A -Tunneled catheter. B - Port catheter.

Both tunneled and port catheters need to be manipulated extremely carefully. After preparing a clean work area, the hands of the nurse/doctor must be washed thoroughly with soap and water, sterile gloves are needed. Flushing with saline solution through the cap/bionector and locking the catheter with heparin solution is required. The dose of heparin varies in function of catheter type and period of closing. ${ }^{16}$

Guidelines for the prevention of complications associated with CVC vary greatly. The widest recommendation is for the use of heparin to prevent occlusion, but some articles suggest that $0.9 \%$ sodium chloride (normal saline) may be as effective. ${ }^{17}$ Other possibilities have been discussed in the literature, such as the injection of $70 \%$ ethanol for a period of two hours, which can eliminate pathogens colonising CVCs, but our center has no experience with this method yet. ${ }^{18}$ Another possibility described is taurolidine lock, which, compared to heparin, is significantly more expensive. ${ }^{19}$

Partial dysfunction of the central venous catheter is common. Fluids can be infused into the catheter, but blood cannot be aspirated. A study with contrast substance and/ or thrombolysis is being undertaken. ${ }^{20}$ We experienced this in case of the 1.4-year-old ALL patient, in whom we could not use her port for one month without knowing what does not work. By the time we programmed her to remove the CVC, it was spontaneously permeabilized, therefore the catheter was maintained.

In neutropenic pediatric oncology patients with central line, infections can occur frequently. ${ }^{21} \mathrm{~A}$ higher infection rate associated with tunneled catheters has been described in the literature as compared to port catheters, while in our study we experienced one infection with tunneled and one infection with totally implanted catheter. ${ }^{22}$
We had one case of thrombosis (14-year-old CC girl), in whom we needed to remove the tunneled catheter and initialized low-weight heparin treatment for 3 months. The literature indicates that Factor VIII activity may be used to stratify critically ill children based on their risk for catheter-related thrombosis, however we do not perform these tests as a routine. ${ }^{23}$

Fever in a patient with a CVC can have a common cause, or it may be due to a catheter-associated bloodstream infection. Catheter-associated infections may lead to additional complications such as sepsis, septic shock, or septic complications including suppurative thrombophlebitis, endocarditis, osteomyelitis and others. Early appropriate antibiotic therapy has been shown to improve outcome. ${ }^{24}$

A multicenter study concluded that there was no benefit in administering antibiotics before the insertion of CVCs to prevent Gram positive catheter-related infections, however flushing and locking CVCs with a combined antibiotic and heparin solution seems to decrease Gram positive catheter-related sepsis. ${ }^{25}$ Another group of feared incidents are multidrug-resistant Gram negative bacterial infections in neutropenic oncologic patients. ${ }^{26}$

The failure of central venous access devices (CVADs) results in interrupted medical treatment, increased morbidity and mortality, and prolonged hospitalization for the patient. $^{27}$

Tunneled catheters may be maintained for a maximum period of 9 months, port catheters for 2-4 years, and this period varies largely, depending on how the patient tolerates it. In our center we experienced 2 cases of necrosis, because of which the port catheters were removed. Some port removals are complicated by CVC fragmentation, especially in case of CVC caliber $<6 \mathrm{Fr}$ and/or $>2$ years since insertion. ${ }^{28}$ 


\section{CONCLUSIONS}

Vein punctures in children cause both physical and emotional pain. The peripheric administration of chemotherapy has an irritating effect on the veins, and these two facts can justify the use of CVC in pediatric oncology. Both tunneled and port catheters need to be manipulated extremely carefully, especially in case of a neutropenic patient, because infections may lead to additional complications such as sepsis, septic shock or other septic complications. The use of central lines in pediatric hemato-oncology is accessible and benefic not only for increasing patient comfort, but also to ease the nurses' work, who are often overburdened.

\section{CONFLICT OF INTEREST}

Nothing to declare.

\section{ACKNOWLEDGMENTS}

We wish to send our special thanks to the University of Medicine and Pharmacy of Tîrgu Mureș and the staff of our Clinic, both nurses and physician colleagues.

\section{ABBREVATIONS}

ALCL anaplastic large cell lymphoma

ALL acute lymfoblastic leukemia

AML acute myeloblastic leukemia

CC colon carcinoma

CVC central venous catheter

CVP central venous pression

DSRCT desmoplastic small round cell tumor

FFP fresh frozen plasma

HL Hodgkin lymphoma

NB Neuroblastoma

NHL Non-Hodgkin Lymphoma

po peroral

WT Wilms tumor

\section{REFERENCES}

1. Fadoo Z, Nisar MI, Iftikhar R, et al. Peripherally Inserted Central Venous Catheters in Pediatric Hematology/Oncology Patients in Tertiary Care Setting: A Developing Country Experience. J Pediatr Hematol Oncol. 2015;37(7):e421-423.

2. Orgel E, Ji L, Pastor W. Infectious morbidity by catheter type in neutropenic children with cancer. Pediatr Infect Dis J. 2014;33(3):263-6.

3. Moureau N, Chopra V. Indications for peripheral, midline and centralcatheters: summary of the MAGIC recommendations. $\mathrm{Br} J$ Nurs. 2016;25(8):S15-24.

4. Woodley-Cook J, Amaral J, Connolly B. Do children without a known bleeding tendency eat undergoing PICC placement require coagulation laboratory testing? Pediatr Radiol. 2015;45(5):727-735.

5. Shenep MA, Tanner MR, Sun Y, et al. Catheter-Related Complications in Children With Cancer Receiving Parenteral Nutrition: Change in Risk Is Moderated by Catheter Type. J Parenter Enteral Nutr. 2016;pii: 0148607115624087
6. Giordano P, Saracco P, Grassi M, et al. Recommendations for the use of long-term central venous catheter (CVC) in children with hematooncological disorders: management of CVC-related occlusion and CVCrelated thrombosis. On behalf of the coagulation defects working group and the supportive therapy working group of the Italian Association of Pediatric Hematology and Oncology (AIEOP). Ann Hematol. 2015;94(11):1765-1776.

7. Ullman AJ, Marsh N, Mihala G, Cooke M, Rickard CM. Complications of Central Venous Access Devices: A Systematic Review. Pediatrics. 2015;136(5):e1331-1344.

8. Rinke ML, Chen AR, Bundy DG, et al. Implementation of a central line maintenance care bundle in hospitalized pediatric oncology patients. Pediatrics. 2012;130(4):e996-e1004.

9. Primic-Zakelj M. Cancer epidemiology. In Scrijvers D, Senn HJ, Mellstedt $\mathrm{H}$, Zakotnk B ( eds) European Society of Medical Oncology Handbook of Cancer Prevention. Informa Healthcare 2008:1- 28

10. Ahn S, Chung JH. Proper tip position of central venous catheter in pediatric patients. J Vasc Access. 2015;16(5):399-402.

11. Morello FP, Donaldson JS, Saker MC, Norman JT. Air embolism during tunneled central catheter placement performed without general anesthesia in children: a potentially serious complication. J Vasc InterV Radiol. 1999;10(6):781-784.

12. Engstrom BI, Horvath JJ, Stewart JK, et al. Tunneled internal jugular hemodialysis catheters: impact of laterality and tip position on catheter dysfunction and infection rates. J Vasc Interv Radiol. 2013;24(9):1295-1302.

13. Maki DG, Stolz SM, Wheeler S. Prevention of central venous catheterrelated bloodstream infection by use of an antiseptic-impregnated catheter. A randomized, controlled trial. Ann Intern Med. 1997;127(4):257-266.

14. Brown-Smith JK, Stoner MH, Barley ZA. Tunneled catheter thrombosis: factors related to incidence. Oncol Nurs Forum. 1990;17(4):543-549.

15. Forauer AR, Theoharis CG, Dasika NL. Jugular vein catheter placement: histologic features and development of catheter-related (fibrin) sheaths in a swine model. Radiology. 2006;240(2):427-434

16. Cesaro S, Tridello G, Cavaliere M, et al. Prospective, randomized trial of two different modalities of flushing central venous catheters in pediatric patients with cancer. J Clin Oncol. 2009;27(12):2059-2065.

17. Bradford NK, Edwards RM, Chan RJ. Heparin versus $0.9 \%$ sodium chloride intermittent flushing for the prevention of occlusion in long term central venous catheters in infants and children. Cochrane Database Syst Rev. 2015;11:CD010996.

18. Schoot RA, van Ommen $\mathrm{CH}$, Stijnen $\mathrm{T}$, et al. Prevention of central venous catheter-associated bloodstream infections in paediatric oncology patients using 70\% ethanol locks: A randomised controlled multi-centre trial. Eur J Cancer. 2015;51(14):2031-2038.

19. Handrup MM, Møller JK, Schrøder H. Central venous catheters and catheter locks in children with cancer: a prospective randomized trial of taurolidine versus heparin. Pediatr Blood Cancer. 2013;60(8):1292-1298.

20. Keidan I, Ben-Menachem E, Berkenstadt H, et al. A Simple Diagnostic Test to Confirm Correct Placement of Dysfunctional Central Venous Catheters Before Chemotherapy in Children. J Pediatr Hematol Oncol. 2016;38(1):78-80.

21. Orgel E, Ji L, Pastor W. Infectious morbidity by catheter type in neutropenic children with cancer. Pediatr Infect Dis J. 2014;33(3):263-6.

22. Zachariah M, Al-Yazidi L, Bashir W, et al. Spectrum of external catheterrelated infections in children with acute leukemia - Single-center experience. J Infect Public Health. 2014;7(1):38-43.

23. Faustino EV, Li S, Silva CT, et al. Factor VIII May Predict Catheter-Related Thrombosis in Critically III Children: A Preliminary Study. Pediatr Crit Care Med. 2015;16(6):497-504.

24. Brennan C, Wang V. Management Of Fever And Suspected Infection In Pediatric Patients With Central Venous Catheters. J Pediatr Emerg Med Pract. 2015;12(12):1-17.

25. van de Wetering MD, van Woensel JB, Lawrie TA. Prophylactic antibiotics for preventing Gram positive infections associated with long-term central venous catheters in oncology patients. Cochrane Database Syst Rev. 2013;11:CD003295.

26. Costa Pde O, Atta EH, Silva AR. Infection with multidrug-resistant gramnegative bacteria in a pediatric oncology intensive care unit: risk factors and outcomes. J Pediatr (Rio J). 2015;91(5):435-41.

27. Ullman AJ, Marsh N, Mihala G, et al. Complications of Central Venous Access Devices: A Systematic Review. Pediatrics. 2015;136(5):e1331-1344.

28. Teague WJ, Fouad D, Munro FD. Complicated vascular access port removals: incidence, antecedents and avoidance. Pediatr Surg Int. 2015;31(9):859-64. 